\title{
Estimated glomerular flltration rate is associated with bone fragility in the elderly
}

M. João Gonçalves ${ }^{1}$, A. Rodrigues ${ }^{1}$, J. Caetano-Lopes ${ }^{1}$, A. Lopes ${ }^{1}$, B. Vidal ${ }^{1}$, E. Raquel ${ }^{1}$, M. Sarmento², F. Vaz $^{3}$, J. Monteiro², J. E. Fonseca1 ${ }^{1}$, H. Canhão ${ }^{1}$

${ }^{1}$ Rheumatology Research Unit, Instituto de Medicina Molecular, ${ }^{2}$ Orthopedics department, Santa Maria Hospital, ${ }^{3}$ Mechanics Engineering Department, Instituto Superior Tecnico, Lisbon

\section{Introduction}

- Osteoporosis is frequently associated with renal disease, namely due to the bone metabolism disturbances caused by secondary hyperparathyroidism of chronic kidney disease (CKD). The increased risk of fragility fractures is well demonstrated in patients with end-stage renal disease (ESRD). There is recent evidence that bone pathological changes start early in the course of CKD[1,2]. Given the high prevalence of both osteoporosis and CKD in older adults, it is of the utmost public health relevance to be able to assess fracture risk in this population.

\section{Objectives}

- Our aim is to evaluate whether CKD, without criteria of ESRD, is associated with bone fragility. Bone fragility was assessed considering history of fragility fracture events, 10 year risk for major osteoporotic fractures and hip fractures (FRAX), biochemical bone turnover markers (P1NP and CTX) and mechanical testing to determine bone stiffness.

\section{Methods}

-We studied patients admitted for total hip replacement surgery. They were asked for clinical data and blood samples. Blood biomechanical studies were performed and a bone cylinder was drilled from their femoral epiphyses. Glomerular filtration rate (GFR) was estimated using Cockcroft-Gault formula; we excluded patients where the application of the formula was inadequate. We also excluded from the analysis patients with ESRD, with eGFR $\leq 15 \mathrm{ml} /$ minute or previous need of renal replacement therapy.

\section{Results}

We included 111 patients. Mean age was $74.31 \pm 10.03,70 \%$ of the subjects were female, $98 \%$ Caucasian.

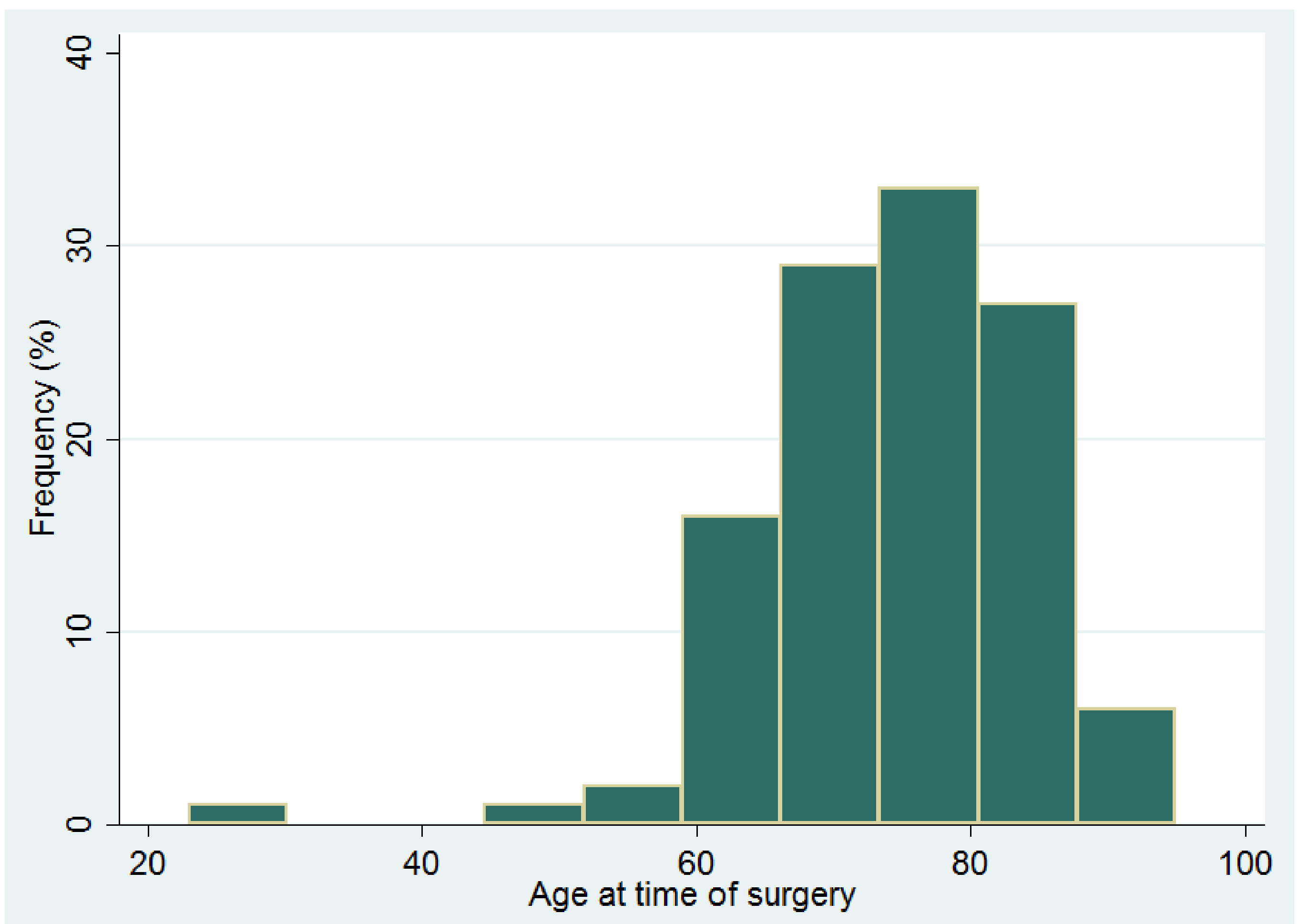

Fig 1 - Distribuition of age at time of total hip replacement

More than half of the subjects (58.1\%), had Chronic Kidney Disease. In the graphic below, the distribuition according to the stages of CKD.

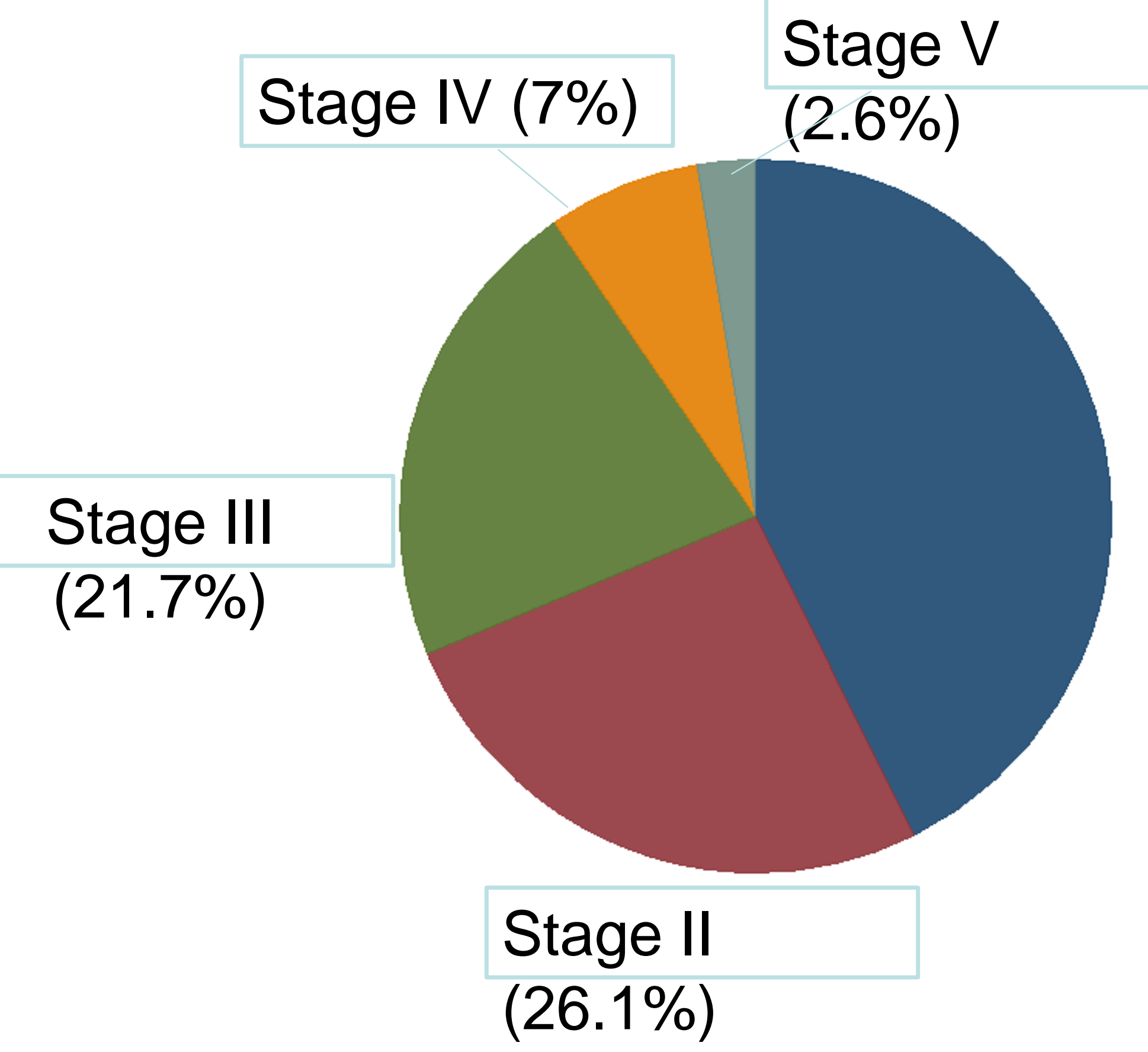

Fig. 2: Frequency (\%) of patients in defferent stages of CKD.

Fragility fracture had an inverted relation with eGFR ( $p=0.023, r=-0.08)$.

Number of fragility fractures

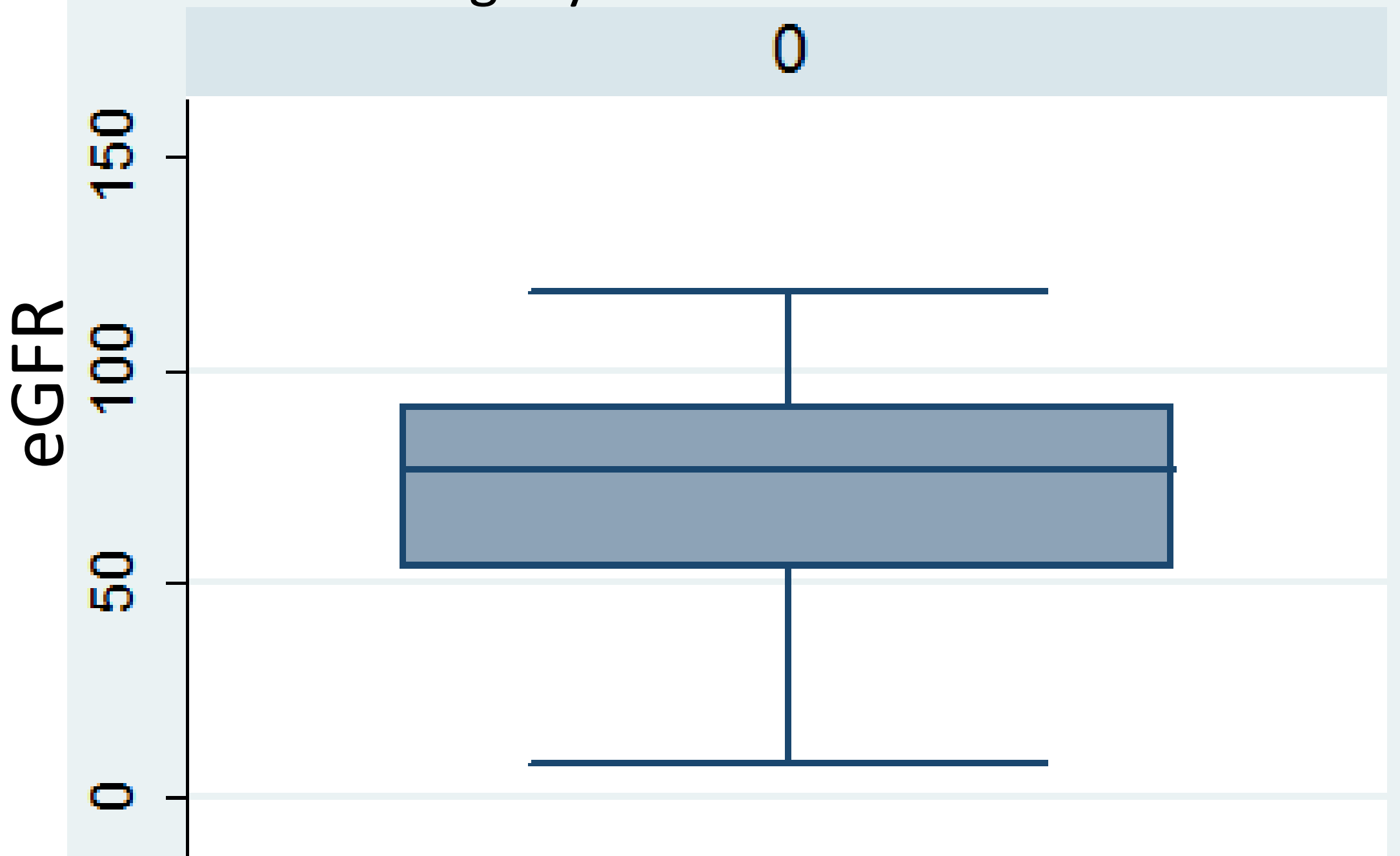

Fig. 3: Mean eGFR in patient with or without fragility fractures
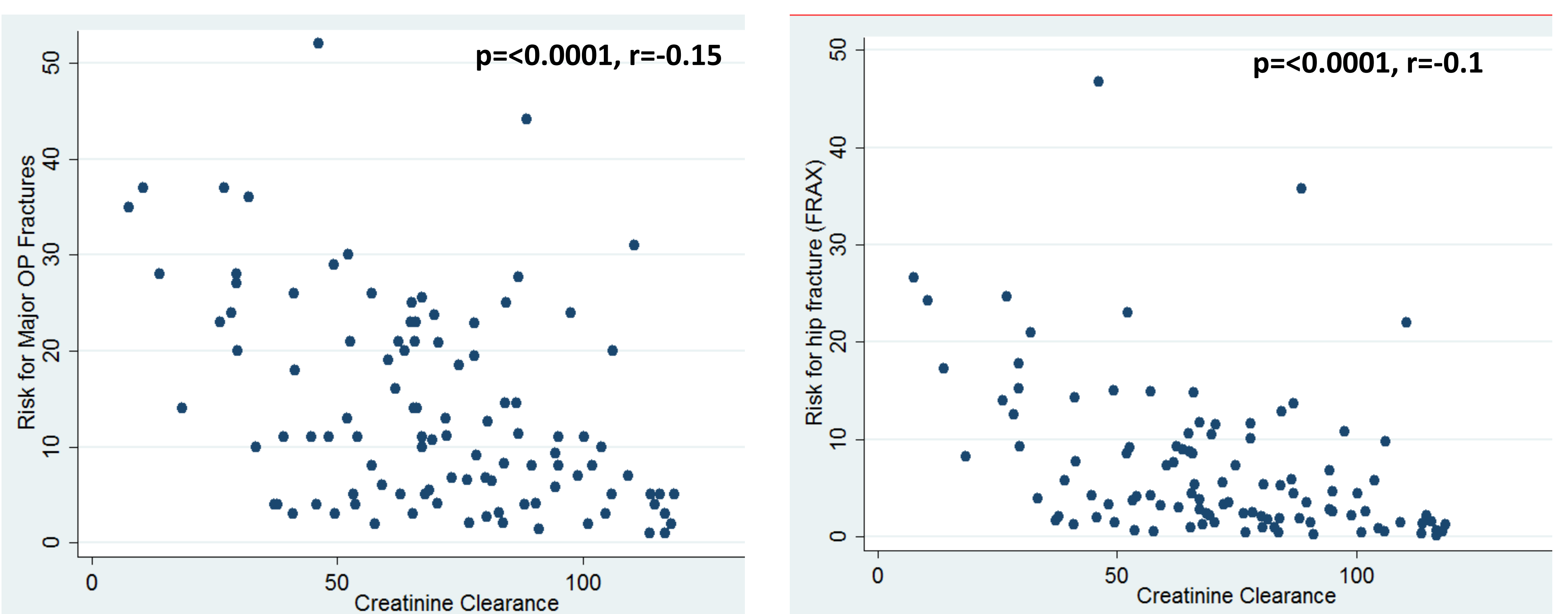

Fig. 4 - The clinical score FRAX for hip and major osteoporotic fractures was inverted related with eGFR.

The biomarker CTX I, a molecule released during the resorption of demineralised organic type I collagen matrix, showed an inverted relation with eGFR ( $p=0.003, r=-0.08)$.

P1NP $(r=-1.33, p=0.056)$ and bone stiffness $(r=2.42, p=0.073)$ showed a trend for association with eGFR.

All analyses were adjusted to age and sex.

\section{Conclusion}

-Renal impairment in early stages, measured by eGFR, was associated with increased bone fragility assessed by fracture events, FRAX, bone turnover biomarkers and biomechanics parameters, after adjustment to age and sex.

- Improving the understanding of bone metabolism disturbances in earlier stages of chronic renal disease might prevent the development of clinical bone disease in these patients. 